\title{
Regional Brain Activity during Different Paradigms of Mental Rotation in Healthy Volunteers: A Positron Emission Tomography Study
}

\author{
Guy Vingerhoets, ${ }^{*}$ Patrick Santens, $\dagger$ Koenraad Van Laere, $\ddagger$ Philippe Lahorte, $\ddagger \S$ \\ Rudi A. Dierckx, $\$$ and Jacques De Reuck $\dagger$ \\ *Laboratory of Neuropsychology, Ghent University, De Pintelaan 185, B-9000 Ghent, Belgium; $\dagger$ Department of Neurology and $\ddagger$ Division \\ of Nuclear Medicine, Ghent University Hospital, De Pintelaan 185, B-9000 Ghent, Belgium; and \$Laboratory for Subatomic \\ and Radiation Physics, Ghent University, Proeftuinstraat 86, B-9000 Ghent, Belgium
}

Received May 22, 2000; published online December 21, 2000

Positron emission tomography (PET) was used to observe changes in regional cerebral blood flow (rCBF) in 10 right-handed healthy volunteers performing two paradigms of mental rotation. In one paradigm, subjects mentally rotated a single alphanumeric stimulus to determine whether it was shown in a normal or mirror-image position. In a second paradigm, subjects mentally rotated and compared pairs of figurative stimuli to determine whether the stimuli were identical or mirror-images. In both paradigms, rCBF was compared with a control task that used identical stimuli, but required no mental rotation. Mental rotation of single alphanumeric stimuli engendered activation in the primary somatomotor area in the left precentral gyrus. Mental rotation of paired figures engendered activation in the left superior parietal lobule and the right frontal medial gyrus. A deactivated area was located in the medial part of the left superior frontal gyrus. Comparison of both paradigms revealed that the left gyrus precentralis was activated significantly during the alphanumeric condition and that the left gyrus lingualis was significantly activated during the paired figures condition. Motor processes may be an inherent part of every mental rotation but the type of motor involvement appears strongly dependent on the specific task or the specific stimuli. Similar paradigms, designed to isolate the same cognitive function, in the same subjects, using the same imaging technology and methodology, but differing only in stimulus material, lead to different areas of neural activation. Task specificity determines the most significant changes in cerebral blood flow in different mental rotation paradigms. $\odot 2001$ Academic Press

Mental rotation involves cognitive spatial transformations of imagined stimuli, and is an important cognitive method in spatial reasoning and problem solving. In the typical experimental design, subjects judge whether pairs of visual stimuli are identical or differ- ent. Usually these stimuli consist of angular multiarmed cubes (Shepard and Metzler, 1971). The two stimuli appear in different orientations, and are either identical or are left-right mirror images. The subject's task is to report whether the stimuli are identical or mirror images. Times to make these judgements typically increase with the angle of difference in orientation between the stimuli, as if observers where mentally rotating them into correspondence (Cooper and Shepard, 1973). Behavioral studies have suggested that people rotate visual mental images much like they would physically manipulate actual objects (Georgopoulos and Pellizzer, 1995; Kosslyn, 1994; Shepard and Cooper, 1982).

The neural basis of mental rotation remains poorly understood. Lesion data suggest that posterior damage, especially to the parietal lobes, plays an important role in mental rotation, and some studies pointed more to right hemispheric involvement (Ditunno and Mann, 1990), whereas others associated impairments in mental rotation with left hemispheric damage (Mehta and Newcombe, 1991). Neural mechanisms underlying mental rotation have been studied by a number of functional imaging studies, and most studies are consistent with the view that mental rotation is not confined to a single cerebral location, but is performed by a number of processes that are being carried out in different parts of the brain. A neural model for the mental rotation of Shepard and Metzler stimuli based on functional magnetic resonance imaging (MRI) was proposed by Cohen et al. (1996) and included the frontal eye fields (responsible for oculomotor function in scanning the complex visual images), the extrastriate visual regions of the superior parietal lobule (responsible for the bulk of the computation for the mental rotation), and the parieto-occipital border (a possible motion-sensitive area believed to be engaged as a processing center for computation based on object motion). In contrast with earlier findings that demonstrated predominant right hemispheric frontal and parietal 
activity during mental rotation in a ${ }^{133}$ Xenon regional cerebrography study (Deutsch et al., 1988), Cohen et al. (1996) found little evidence for a lateralization of the cortical activity and concluded that hemispheric dominance in mental rotation is quite variable. A functional MRI study by Carpenter et al. (1999) demonstrated a linearly increasing activation in bilateral intraparietal sulcal regions with larger angular disparities between the Shepard-Metzler stimulus pairs. This finding supports the view that the parietal region is particularly implicated in computing spatial coordinates during mental rotation. Additional activation in the fusiform gyrus and inferior temporal regions was believed to illustrate processes of object and object-part identification. Modest frontal activation, including the precentral gyrus and sulcus, and the posterior middle frontal gyrus was interpreted by these authors as oculomotor activity associated with planning and executing saccades. Bilateral parietal activation was also demonstrated in other functional MRI studies (Iwaki et al., 1999; Tagaris et al., 1996, 1997; Richter et al., 1997) and in a PET study (Kosslyn et al., 1998) using the same or very similar mental rotation paradigms.

Some studies have experimented with other stimuli and/or paradigms to reveal the neural basis of mental rotation. A PET study using a single alphanumeric stimulus paradigm observed (left) superior parietal lobule and especially (left) inferior parietal lobule activation that was hypothesized to be involved in the active mental transformation of the stimuli (Alivisatos and Petrides, 1997). These investigators noted right frontal activation (Brodmann areas (BA) 45 and 8) that was attributed to increased executive processing and working memory. In addition, right hemispheric activation in the head of the caudate nucleus was hypothesized to be involved with cognitive motion of stimuli in space. With regard to lateralization, Alivisatos and Petrides concluded that during mental rotation activity was more intense in the left parietal cortex. In a similar letter-rotation functional MRI study, Tagaris et al. (1998) detected predominant bilateral activation in the precentral gyrus, intraparietal cortex, and extrastriate visual cortex. An elegant PET study of Harris et al. (2000) used the same alphanumeric mental rotation paradigm as Alivesatos and Petrides (1997), but they used an experimental parametric approach instead of the usual cognitive subtraction technique. The study revealed selective activation in the right posterior parietal lobe, centered on the intraparietal sulcus (BA 7). In agreement with experimental animal and human research, the authors suggest that the intraparietal sulcus and the superior parietal lobule are involved in implementing a variety of visuospatial transformations such as mental rotation. In a functional MRI study, Zacks et al. (1999) described slightly right hemispheric asymmetric activation of the parietal-temporal-occipital junction and substantial left frontal acti- vation in a mental rotation task using human figures. Parsons et al. (1995) used PET to study a task that appears to involve the mental rotation of hands (is the pictured hand a right or a left hand?). Usually this task is solved by remodeling it as a reaching task in which subjects implicitly turn their own hands into correspondence with the pictured stimulus. Activation in frontal motor areas (left supplementary motor and bilateral (although predominantly left) anterior cingulate and superior premotor area), bilateral superior parietal areas, cerebellum, and basal ganglia was recorded. The authors concluded that motor imagery, used in mental transformations of the viewer rather than of the viewed object, was realized through neural systems of movement but did not require the primary sensory or motor cortices. Investigating the neural correlates of mental transformations of the body-in-space, and using a similar hand rotation paradigm in their PET study, Bonda et al. (1995) found significant bilateral activation in the superior parietal lobule (attributed to imagery spatiotemporal projections of the body in space) and in the anterodorsal part of the insula (attributed to higher levels of somatic function). Other significant CBF foci were noted in cortical and subcortical motor structures: the premotor cortex, the cingulate motor areas, the putamen, and the cerebellum that are associated with motor imagery.

Kosslyn and coworkers (Kosslyn, 1994; Kosslyn et al., 1998) elaborated on the seemingly physical and analog characteristics of mental rotation. They argued that "the laws of physics do not prevent a mental image from undergoing instantaneous translation from one position to the next." Evidently, this argument does not provide an explanation as to why people visualize objects rotating through trajectories? With respect to this issue, Kosslyn et al. (1998) suggested that motor processes may play a role in mental rotation. These authors not only expected activation in higher-level motor control areas such as the supplementary motor area, premotor area, and posterior parietal lobe, but they also expected activation in low-level motor areas such as the precentral gyrus. Indeed, mental rotation appears to involve some kind of motion of an imagined movement vector and both real and imagined movements are governed by similar amplitude-accuracy relations (Georgopoulos and Pellizzer, 1995). Kosslyn et al. (1998) compared the mental rotation of (pairs of) hands and the mental rotation of Shepard-Metzler figures in the same volunteers to investigate whether specific stimuli might elicit different systems of neural activity. Mental rotation of Shepard-Metzler figures activated the bilateral inferior and superior parietal lobules and BA 19. Mental rotation of the hands invoked predominantly left hemispheric activation in the precentral gyrus (primary motor cortex), superior and inferior parietal lobules, insula, frontal BAs 6 and 9, and bilateral primary visual cortex. Direct comparison 
of both tasks showed that no areas were activated more in the Shepard-Metzler figures rotation task than in the hands rotation task. Areas that were more activated during hands rotation than during figure rotation included the left hemispheric areas of precentral gyrus, Heschl's gyrus, insula, and the dorsolateral prefrontal cortex. This was interpreted as evidence that, in general, low-level motor processes were only recruited when one mentally rotates hands. Kosslyn et al. (1998) concluded that the method used to mentally rotate stimuli depends on the precise nature of the task or on the specific stimuli and that a least two different mechanisms can be used, one mechanism recruiting processes that relies heavily on motor movements and another mechanism that does not.

A first conclusion that emerges from this overview is that parietal activation, viewed by most authors as the region involved with mental transformation per se, is very often supplemented with activity in frontal and subcortical motor regions. Evidence is getting stronger that transformations of mental images are at least in part guided by motor processes, even in the case of images of abstract objects rather than of body parts (Kosslyn et al., 1998; Wexler et al., 1998; Parsons, 1994; Parsons et al., 1995).

Second, it appears that different paradigms of mental rotation result in somewhat different clusters of cortical activity. Both the kind of stimuli (3-D angular multiarmed Shepard-Metzler cubes, alphanumeric stimuli, human hands, human figures) and the specific task involved (rotating a single stimulus or comparing two rotated stimuli) may result in differences between studies. With regard to the kind of stimuli, it has been suggested that the mental rotation of abstract objects may require less low-level motor processing than the mental rotation of body parts (Kosslyn et al., 1998). With regard to the underlying task, rotation of a single stimulus has the advantage of minimizing eye movements and attentional shifts between paired stimuli, both of which are known to activate superior parietal and superior frontal cortex (Corbetta et al. 1993; Anderson et al., 1994). On the other hand, the mental rotation of a single stimulus requires the comparison against a memorized symbol (as in determining whether an alphanumeric stimulus is mirror-imaged or not) or against a mental body image (as in determining whether the stimulus pictures a right or a left hand). It can also be hypothesized that mental rotation paradigms based on comparison of paired stimuli are more prone to perceptual bias caused by eye movements, attentional shifts, and increased visual complexity of the stimuli. Well-known, over-learned symbols, or body parts (often used in single stimulus mental rotation paradigms) may be more prone to the involvement of physically guided motor processes for the mental manipulation of the stimulus against a preexisting "normal" mental image. People find it eas- ier to mentally rotate body parts or objects that are commonly manipulated with the hands (and maybe also over-learned symbols), in ways that do not violate the normal physical movements of that body part (Parsons, 1987, 1994; Sekiyama, 1982; Wexler et al., 1998).

Third, the critical reader can't help noticing that similar studies using an (almost) identical mental rotation paradigm may report very dissimilar results. This observation suggests the existence of potentially hidden variables that influence regional cerebral activation. Subtle methodological differences between studies, and perhaps also individual differences in cognitive task approach in these generally small-sample studies may contribute to the contradicting findings.

The more modest aim of the present study was to observe changes in regional cerebral blood flow during mental rotation and to evaluate the differences in neural activity triggered by two different paradigms designed to isolate the function of mental rotation in the same volunteers. Both paradigms used in this study require a similar decision (is the stimulus a mirrorimage or not), but differ in the method on how to achieve that decision. In a single alphanumeric paradigm subjects have to rotate and compare a well-known symbol against a preexisting mental image, whereas in the paired stimuli paradigm, subjects have to make a comparison between unfamiliar images. We hypothesized that (1) different paradigms of mental rotation lead to different yet partially overlapping systems of neural activity associated with mental rotation per se, and (2) comparison of the different paradigms reveals the cognitive functions necessary to perform that specific mental rotation task. More in particular we would expect more perceptual bias (resulting in (higher) superior parietal, superior frontal, and occipital activation) in the paired stimuli paradigm over and above the expected mental rotation (superior parietal activation) and some possible higher-level motor processing (activation of supplementary motor and premotor areas). In the single alphanumeric stimulus paradigm we would expect less perceptual bias, but more pronounced (lowlevel) motor activation (precentral gyrus), and possible semantic memory activation over and above mental rotation.

\section{MATERIAL AND METHODS}

\section{Subjects}

Following ethical committee approval and informed consent, 10 members of the Ghent University Hospital community volunteered for this study. All participants (5 women and 5 men) were in good general health without a history of neurological or psychiatric disorders, between 23 and 34 years of age (mean age, 26 years; SD, 3.1 years) and had an average of 16.5 years of formal education (SD: 1.6 years). All subjects were 


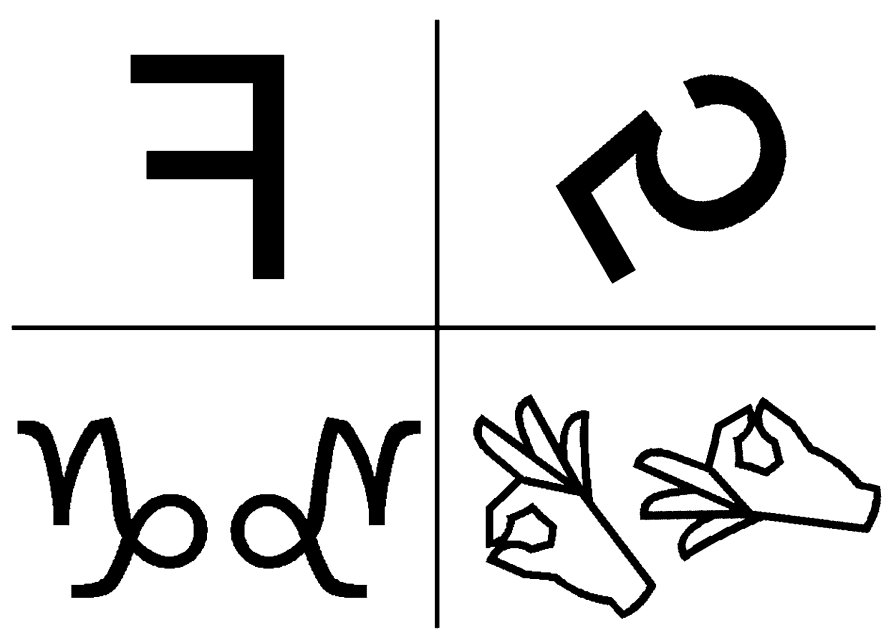

FIG. 1. Illustrations of "normal/same" and "mirror-image" stimuli used in the single alphanumeric paradigm (top) and in the paired figures paradigm (bottom). The left panels show stimuli in the control condition, the right panel illustrates stimuli in the rotation condition.

right-handed, as measured by the Edinburgh Handedness Inventory (Laterality Index: mean \pm SD, $94 \pm$ 8.9\%; range, $75-100 \%$ ). The participants were not aware of the purposes or hypotheses of the experiment until after test completion.

\section{Activation Paradigms}

Subjects were presented with two cognitive paradigms each consisting of two conditions (Fig. 1). The first paradigm (A) was similar to the task used by Alivisatos and Petrides (1997). The stimuli consisted of single alphanumeric characters that were asymmetrical in the horizontal and vertical axes, namely the upper case letters G, F, R and the Arabic numerals 2, 5,7 . In a first (control) condition (A1) each stimulus was presented in either its normal or backward (i.e., mirror image) form. Subjects were instructed to press the button held in the left hand if the stimulus was shown in the mirror image form and to press the button held in the right hand if the stimulus was shown in its normal form. Left- and right-handed responses were balanced over the 2-min acquisition period to avoid disproportionate activation of one motor cortex. In the second (rotation) condition (A2) the same alphanumeric stimuli were used and the subject was again required to make the same judgement on whether the stimulus was shown in its normal or mirror image form. In this condition, however, the stimulus was presented in one of three orientations: $120^{\circ}, 180^{\circ}$, or $240^{\circ}$. The subjects had to mentally rotate the stimulus into the upright position in order to make the correct judgement, again by pressing the hand-held buttons.

The stimuli of the second paradigm (B) consisted of two identical typographic symbols or simple line drawings of hand positions that were presented on the screen. The stimulus on the left was always presented in a "normal" upright position. In a first (control) condition (B1) the stimulus on the right side was presented in either its "normal" orientation (i.e., identical of the left stimulus) or as a mirror image of the left stimulus. Subjects had to decide whether the right figure was shown in its normal or backward form by pressing the hand-held buttons. In a second (rotation) condition (B2) the right-sided stimulus was also rotated over $120^{\circ}, 180^{\circ}$, or $240^{\circ}$ and again subjects had to mentally rotate the stimuli in an upright form to judge the stimulus as a normal or mirror image of the left stimulus by pressing the hand-held buttons.

All stimuli were presented using the Microsoft PowerPoint97 computer program. Each stimulus was shown for three seconds on a computer monitor, the images subtended approximately $13 \times 10 \mathrm{~cm}$. Each stimulus was immediately replaced by the following stimulus. The monitor was mounted from the ceiling and allowed each subject an optimal view while they were lying in a supine position in the scanner. Distance from the screen and visual angles were virtually identical for each subject. Prior to scanning, the tasks were explained and a block of practice trials was offered. Subjects were instructed to respond both as quickly and as accurately as possible and to lie absolutely still during scanning. Each condition lasted $80 \mathrm{~s}$ and was assessed twice in the order A1 A2 B1 B2 A1 A2 B1 B2, which was identical for each subject. All subjects reported that they were able to perform the task within the scanner without difficulty and had a clear and comfortable view to the monitor. No problems were experienced with the pacing of the stimuli. Response times were not recorded because of logistical reasons. There is ample evidence that these paradigms produce the behavioral signature of mental rotation in a diversity of stimuli, namely an increasing response time with larger angular disparity (Cohen et al., 1996; Georgopoulos and Pellizzer, 1995; Kosslyn et al., 1998; Tagaris et al., 1998).

\section{Data Acquisition}

All PET studies were acquired on a Siemens ECAT 951/31 PET scanner with 16 detector rings, allowing reconstruction of 31 consecutive slices along an axial field of view of $10.8 \mathrm{~cm}$ (2-D mode). Axial resolution is $5.7 \mathrm{~mm}$ and transversal resolution $6.4 \mathrm{~mm}$.

In all subjects a transmission scan was performed first by means of a ${ }^{68} \mathrm{Ge} /{ }^{68} \mathrm{Ga}$ ring source to allow subsequent attenuation of emission data.

rCBF estimates during activation were obtained by inhaling $\mathrm{C}^{15} \mathrm{O}_{2}$ at $700 \mathrm{MBq} / \mathrm{min}$. In each of the eight conditions, presentation of the paradigm was started ten seconds before tracer inhalation. Another $10 \mathrm{~s}$ later, approximately at the time the cerebral activity counts increased, the $60 \mathrm{~s}$ data acquisition period was 
started. Between scanning episodes, the subjects remained in their original position with the inhalation mask temporarily removed to reduce discomfort throughout the entire procedure. A 12-min period (approximately six halflifes) was respected between studies to allow decay of the tracer. During this period, subjects were instructed and practice trials were offered.

\section{Image Processing}

Sinogram images were reconstructed using filtered back projection (Hanning filter, cut-off 0.5), with nonuniform transmission correction. Reconstructed images were transferred in Interfile format onto a central PC-based image processing system (HERMES, Nuclear Diagnostics, Sweden) by means of the in-house written MedCon conversion program. Because of improved noise characteristics, the reconstructed data were added for each subject to generate a mean image. Intense nasal cavity and extracerebral scalp activity was masked out of the mean and individual images by means of a set of individual regions-of-interest (ROIs). The images were then smoothed (Butterworth filter 0.9 cycli/cm, order 8 ) and fitted automatically onto a inhouse constructed perfusion database $(n=30)$ positioned in Talaraich and Tournoux coordinates (Talairach and Tournoux, 1988). This fitting procedure was performed with nine parameters (scale, shift, and rotation) using a principal axis transform and mutual information minimization algorithm (fit threshold of 0.50; BRASS, Nuclear Diagnostics, Sweden) (d'Asseler et al., 1999).

\section{Statistical Parametric Mapping}

To apply statistical parametric mapping within SPM96 (Wellcome Department of Cognitive Neurology, Institute of Neurology, London, UK), images were converted into ANALYZE format by means of the same MedCon conversion software. All SPM calculations were performed in Matlab 4.2 (The MathWorks, Natick, MA) on a SUN SPARC 10 computer (Sun Microsystems Europe Inc, Brussels, Belgium). Normalization was performed by applying the transformation parameters of the SPECT perfusion template onto a PET SPM96 template smoothed isotropically with a 14-mm gaussian kernel. This normalization was done using a nine-parameter rigid body transformation (shift, scale, and rotation). No affine (shear) parameters were used because of the relatively poor spatial resolution and noise characteristics of PET data. Differences in global activity between scans were removed by scaling the activity in each pixel proportionally to the global activity. Mean global activity of each scan was adjusted to 50 . The threshold value for gray matter was put at 0.60 and a resulting voxel size of $3 \times 3 \times$ $3 \mathrm{~mm}$ was used. The normalized studies were smoothed with an isotropic 14-mm kernel to account for individual variability in structure-function relation and to improve signal-to-noise ratio (Friston et al., 1995). For determination of significant sites of increased or decreased perfusion, a categorical multistudy, multisubject, multiple condition model was used. Planned comparisons between conditions were performed on a pixel-by-pixel basis by $t$ statistics, generating $\operatorname{SPM}(t)$ maps subsequently transformed to the unit normal distribution $\operatorname{SPM}(Z)$ maps. We investigated areas by using a height threshold corresponding to at least $P<0.01$ and an extent threshold at $P<$ 0.05 . No correction for multiple comparisons was made, which implies that surviving areas were considered to be meaningful only in the presence of preexisting anatomical or physiological hypotheses.

The key comparison of this study was between the rotation and the control tasks for each paradigms. Both tasks required the volunteers to encode the visual images, to compare them, and to make a decision. Differential activity in the rotation task compared to the control task will reflect the rotation process per se. To determine areas of activation during mental rotation, the control tasks were subtracted from their respective rotation conditions (A2-A1 and B2-B1). Areas of deactivation were determined by subtracting the rotation condition from the control condition (A1-A2 and B1B2). In order to address the question whether a specific distribution of activation might be related to common involvement of mental rotation in both tasks, the contrast $(\mathrm{A} 2+\mathrm{B} 2)-(\mathrm{A} 1+\mathrm{B} 1)$ was performed. (A1 + $\mathrm{B} 1)-(\mathrm{A} 2+\mathrm{B} 2)$ was calculated to reveal deactivated areas. Our second aim was to investigate the differential effect of different rotation paradigms. Several strategies can be pursued. First, we can make a global comparison between the paradigms. To investigate this we simply subtracted the paired stimuli paradigm tasks (control and rotation) from the alphanumeric paradigm tasks (control and rotation), and vice versa $[(\mathrm{A} 1+\mathrm{A} 2)-(\mathrm{B} 1+\mathrm{B} 2)$ and $(\mathrm{B} 1+\mathrm{B} 2)-(\mathrm{A} 1+\mathrm{A} 2)]$. This gives a general idea of the additional activation needed to perform one paradigm over the other. Second, we can directly compare the activation during the mental rotation task between the paradigms (B2 - A2 and A2 - B2). Third, we can first subtract out the control conditions from their respective rotation conditions before subtracting them [(A2 - A1) - (B2 - B1)] and $[(\mathrm{B} 2-\mathrm{B} 1)-(\mathrm{A} 2-\mathrm{A} 1)]$. The coordinates of the single most-activated pixel in each normalized Montreal Neurological Institute template area were corrected for the equivalent coordinates in the Talairach and Tournoux atlas brain (1988) that was used to identify the activated areas. We used the Meyer-Lindenberg conversion (with $X^{\prime}=0.88 X-0.8 ; Y^{\prime}=0.97 Y-$ 3.32; and $Z^{\prime}=0.05 Y+0.88 Z-0.44$ ) described by $\mathrm{M}$. Brett (http://www.mrc-cbu.cam.ac.uk/Imaging/mnispace. $\mathrm{html}$ ) because this method is best suited for the SPM96 coordinates. 
A

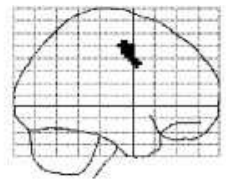

B

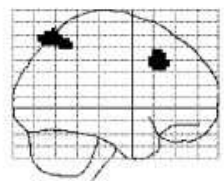

C

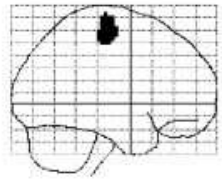

D

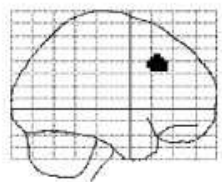

E

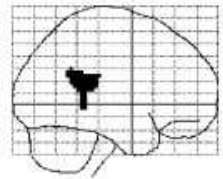

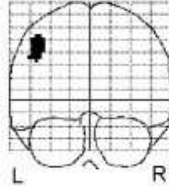
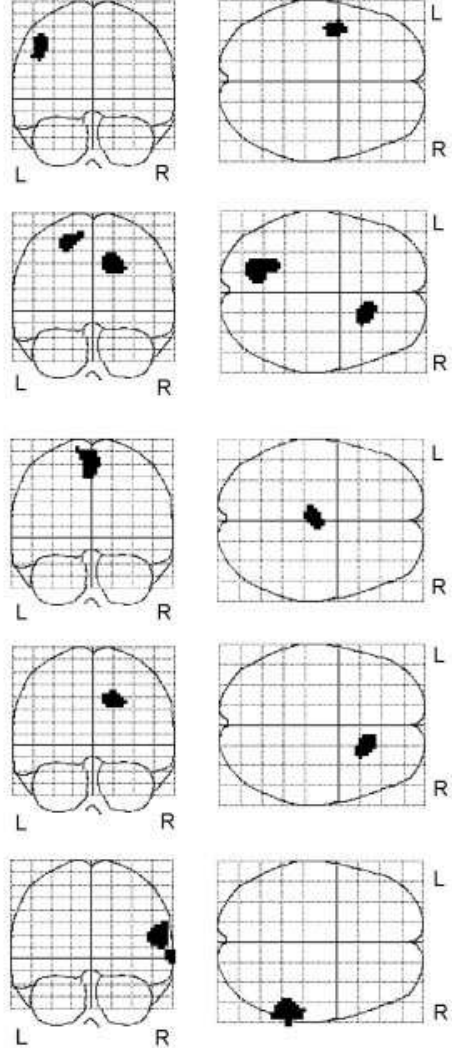

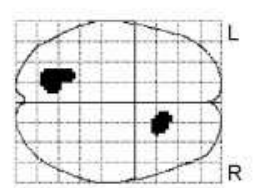

G

H

I
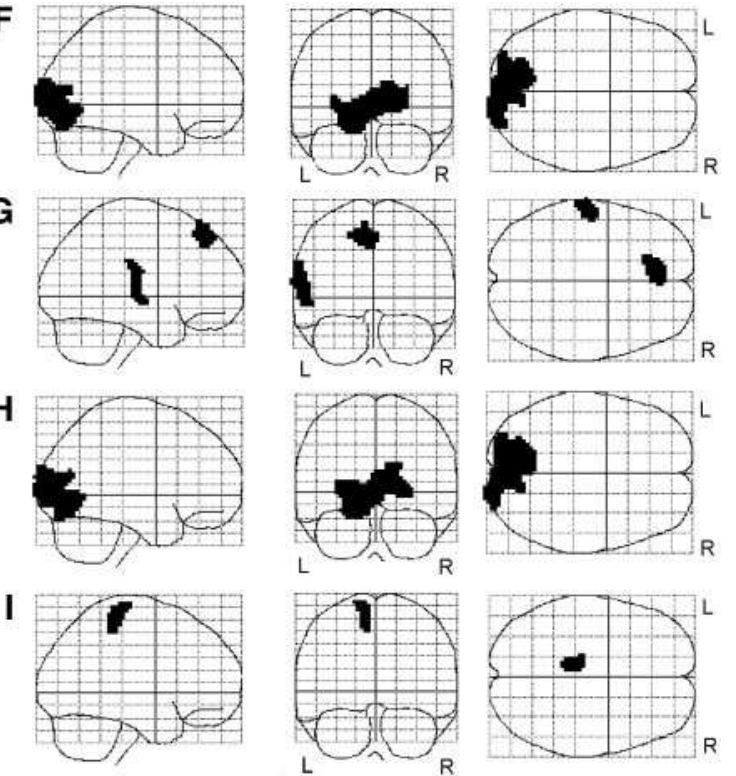

FIG. 2. Activated areas during mental rotation of single alphanumeric stimuli (A) and during mental rotation of paired figures (B). Deactivation during mental rotation of paired figures (C). Activation (D) and deactivation (E) during mental rotation of both paradigms combined. Additional activation during the paired figures paradigm (F) and during the alphanumeric paradigm (G). Additional activation during the paired figures rotation task $(\mathrm{H})$ and the alphanumeric rotation task (I).

\section{RESULTS}

Mean accuracy score for every condition was over 90\% (range 90.5-99.5\%) implying low error rates. A $4 \times 2$ repeated measures multivariate analysis of variance (MANOVA) with condition (A1, A2, B1, and B2) and time (first measurement, second measurement) as within-subjects factors was performed to investigate condition and learning effects. The MANOVA revealed a significant effect of condition (Hotelling's $T^{2}[3,7]=$ $10.71, P=0.005$ ). Posthoc comparisons indicated that the mental rotation of paired stimuli was significantly more difficult than both control tasks, but not significantly more difficult than the mental rotation of single alphanumeric stimuli. There was no significant effect of time, nor a significant condition by time interaction effect.

Increased neural activity during the single alphanumeric stimuli paradigm that can be attributed to mental rotation was located in the primary somatomotor area in the left precentral gyrus (BA 4) (Fig. 2A and Table 1). No areas of deactivation were found for this paradigm. Activated areas during the paired figurative stimuli paradigm included the left superior parietal lobule (BA 7) and the frontal oculomotor field in the right medial part of the superior frontal gyrus (BA 8/32) (Fig. 2B and Table 1). A deactivated area during this paradigm was located in the supplementary motor area in the medial part of the left superior frontal gyrus (BA 6) (Fig. 2C and Table 1). Comparison of all mental rotation tasks versus all control tasks revealed an activation of the oculomotor area of the right medial part of the superior frontal gyrus (BA 8/32) and a deactivation of the auditory association area of the right superior temporal gyrus (BA 22) (Figs. 2D and $2 \mathrm{E}$ and Table 1).

Comparison of both paradigms revealed that the left gyrus lingualis (BA 17/18) was significantly more activated during the paired figures rotation task (and the entire figures rotation paradigm) than during the alphanumeric task(s) (Figs. $2 \mathrm{H}$ and $2 \mathrm{~F}$ and Table 1). The alphanumeric paradigm engendered more activity in the superior frontal oculomotor area (BA 8) and the middle to superior temporal gyrus (BA 21) than did the paired stimuli paradigm (Fig. $2 \mathrm{G}$ and Table 1). The left gyrus precentralis (BA 4) was significantly more activated in the alphanumeric rotation task than in the paired figures rotation task (Fig. 2I and Table 1). Subtracting out the control conditions before subtracting 
TABLE 1

Areas of Significant (De)activation Following Subtraction of Conditions

\begin{tabular}{|c|c|c|c|c|c|c|}
\hline & Activation & Deactivation & $X\left(X^{\prime}\right)^{a}$ & $Y\left(Y^{\prime}\right)^{a}$ & $Z\left(Z^{\prime}\right)^{a}$ & $Z$ score \\
\hline \multicolumn{7}{|l|}{ Areas (de)activated by mental rotation } \\
\hline \multicolumn{7}{|l|}{ Single alphanumeric stimulus paradigm } \\
\hline Left precentral gyrus (BA 4) (A2-A1) & - & & $-42(-37.8)$ & $-6(-9.1)$ & $48(41.5)$ & 3.85 \\
\hline \multicolumn{7}{|l|}{ Paired figurative stimuli paradigm } \\
\hline Left superior parietal lobule (BA 7) (B2-B1) & • & & $-15(-14.0)$ & $-72(-73.2)$ & $63(51.4)$ & 4.14 \\
\hline Right medial part of superior frontal gyrus (BA 8/32) & & & & & & \\
\hline (B2-B1) & • & & $18(15.0)$ & $21(17.0)$ & 39 (34.9) & 4.14 \\
\hline Medial part of superior frontal gyrus (BA 6) (B1-B2) & & - & $0(-0.8)$ & $-18(-20.8)$ & $63(54.1)$ & 3.79 \\
\hline \multicolumn{7}{|l|}{ Both paradigms combined: all rotations versus all controls } \\
\hline \multicolumn{7}{|l|}{ Right medial part of superior frontal gyrus (BA 8/32) } \\
\hline$[(\mathrm{A} 2+\mathrm{B} 2)-(\mathrm{A} 1+\mathrm{B} 1)]$ & - & & $18(15.0)$ & $21(17.0)$ & 39 (34.9) & 4.67 \\
\hline \multicolumn{7}{|l|}{ Right superior temporal gyrus (BA 22) } \\
\hline$[(\mathrm{A} 1+\mathrm{B} 1)-(\mathrm{A} 2+\mathrm{B} 2)]$ & & $\bullet$ & 57 (49.4) & $-42(-44.1)$ & $18(13.3)$ & 4.06 \\
\hline \multirow{2}{*}{\multicolumn{7}{|c|}{$\begin{array}{l}\text { Comparison of paradigms } \\
\text { Additional activation during paired figures }\end{array}$}} \\
\hline & & & & & & \\
\hline Lingual gyrus (BA 17) $[(\mathrm{A} 1+\mathrm{A} 2)-(\mathrm{B} 1+\mathrm{B} 2)]$ & - & & $-6(-6.1)$ & $-87(-87.7)$ & $0(-4.8)$ & 6.08 \\
\hline \multicolumn{7}{|l|}{ Additional activation during alphanumeric paradigm } \\
\hline \multicolumn{7}{|l|}{ Left middle temporal gyrus (BA 21) } \\
\hline$[(\mathrm{B} 1+\mathrm{B} 2)-(\mathrm{A} 1+\mathrm{A} 2)]$ & • & & $-57(-51.0)$ & $-15(-17.9)$ & $-3(0.4)$ & 3.36 \\
\hline Superior frontal gyrus (BA 8) & & & & & & \\
\hline$[(\mathrm{B} 1+\mathrm{B} 2)-(\mathrm{A} 1+\mathrm{A} 2)]$ & • & & $-3(-3.4)$ & $39(34.5)$ & 45 (41.1) & 3.30 \\
\hline \multicolumn{7}{|l|}{ Figures rotation minus alphanumeric rotation } \\
\hline Lingual gyrus (BA 17) (B2 - A2) & • & & $-9(-8.7)$ & $-69(-70.3)$ & $-3(-6.5)$ & 5.56 \\
\hline \multicolumn{7}{|l|}{ Alphanumeric rotation minus figures rotation } \\
\hline Left precentral gyrus (BA 4) (A2 - B2) & • & & $-12(-11.4)$ & $-27(-29.5)$ & $72(61.6)$ & 3.91 \\
\hline
\end{tabular}

${ }^{a}$ The stereotaxic coordinates are expressed in $\mathrm{mm} . X$, medial-to-lateral distance relative to the midline (positive $=$ right); $Y$, anteriorposterior distance relative to the anterior commissure (positive $=$ anterior); $Z$, superior-inferior distance relative to the anterior commissureposterior commissure line (positive $=$ superior). $X, Y$, and $Z$ values are the MNI-SPM96 coordinates of the single most-activated pixel, whereas the $X^{\prime}, Y^{\prime}$, and $Z^{\prime}$ values represent the equivalent coordinates for the Talairach and Tournoux atlas brain (1988) following Meyer-Lindenberg correction for anatomical differences between the MNI and Talairach brains.

the rotation conditions did not reveal any significant activation.

\section{DISCUSSION}

The relative paucity of activated areas in our study is in contrast with the large number of activated regions in most other studies. For the most part this difference can be explained by the specific and voxel based method used to analyze our data. SPM is a relatively conservative technique with minimal Type I errors (false positives) but a higher possibility of Type II errors (false negatives). In our experiments, only two repeats of each condition were measured, which may decrease sensitivity of the procedure. The investigation of two paradigms in one single PET session puts a practical limit on the total number of repeats that is feasible. Only Kosslyn et al. (1998) used SPM95, but instead of defining the significant voxels by the standard combined intensity/extent thresholding and Gaussian field theory (Friston et al., 1995) the authors arbitrarily defined $Z$ thresholds higher than 3 as significant when a priori hypothesis was available. No mention was made in their work on the actual threshold values. Region-of-interest based approaches used in other work (Carpenter et al., 1999) are known to be subject to false positive errors.

Analysis of our behavioral data suggests that although we did not randomize conditions, there was no significant improvement in accuracy score between the first and second measurement within conditions and there were no significant improvements in performance between the control and target conditions of the first and second paradigm. A possible learning effect reflected by decreased response times cannot be excluded because response times were not measured.

\section{Single Alphanumericals}

It appears from our data that different brain regions can be activated for different kinds of mental rotation tasks or stimuli. In the single alphanumeric stimulus paradigm a significant activation of the left primary motor cortex was found. Precentral gyrus activation has been reported in several mental rotation paradigms. Mental rotation of single letters elicited (among other areas) bilateral activation of the gyrus precentralis in the study of Tagaris et al. (1998). Three of six right-handed subjects showed activation of the left motor area $(\mathrm{BA} 4,6)$ and/or supplementary motor area 
during mental rotation of simplified Shepard-Metzler stimuli in the functional MRI study of Iwaki et al. (1999). Right frontocentral activation was found in the event-related potentials study of Inoue et al. (1998) using paired abstract 2-D stimuli. Kosslyn et al. (1998) found left precentral gyrus activation in a paradigm using paired drawings of human hands. In combination with activation of the left premotor area 6 and the superior parietal area 7 and encouraged by animal research (Rizzolati et al., 1988; Sakata and Taira, 1994; Taira et al., 1990), Kosslyn et al. (1998) associated these areas with the preparation of hand movements, i.e., the integration of sensory input and motor output to guide grasping behavior. Using the same paradigm as in our study however, Alivisatos and Petrides (1997) reported activation in multiple and bilateral brain areas, some of which are associated with motor processing, but not in the precentral gyrus itself, and Harris et al. (2000) did not find any frontal (motor) activation at all.

It appears that although the presence of precentral gyrus involvement in paradigms of mental rotation is not inexplicable, it is certainly not always found. Moreover, the two studies that used a very similar paradigm as in this study did not report precentral gyrus activation (Alivesatos and Petrides, 1997; Harris et al., 2000). Perhaps there is an alternative explanation. In the Alivesatos and Petrides (1997) and Harris et al. (2000) studies, subjects responded by pressing one of two buttons with one of two fingers of the right hand. In the present study a right-hand button is consistently used in response to the non-mirror visual stimulus, and a left-hand button in response to the mirror image. The left precentral gyrus activation might reflect an improved (learning) response to the more familiar stimulus, not necessarily related to mental rotation (Iacoboni et al., 1998). This possible motor preparation bias seems unlikely for two reasons. First, the familiarity effect would certainly be greater in the control condition (A1) using upright alphanumeric stimuli only (which is also repeated twice), that is later subtracted from the mental rotation condition (A2), and yet we find an activated (positive) left precentral area. Second, a similar motor preparation bias (normal = right hand) can also be assumed for the paired figures paradigm were no such activation was found. Subtracting the paired figures rotation from the alphanumeric rotation still leaves a significant left precentral gyrus activity (Table 1 and Fig. 2I). We conclude that our results suggest the contribution of low-level frontal motor processes associated with the mental rotation of single alphanumeric stimuli. The expected activation of parietal areas was not significant in this paradigm. Note, however, that the superior parietal (BA 7) and frontal oculomotor (BA 8) areas activated in the paired figures rotation are no longer significant when this condition is subtracted from the alphanumeric rotation (Table 1 and Fig. 2H), implying that they might also have been involved in the mental rotation of alphanumeric stimuli, but failed to reach significance.

\section{Paired Figures}

In our paired figurative stimuli paradigm, activation of the left superior parietal lobule and right medial part of the superior frontal gyrus was found. Activity in the superior parietal lobule (BA 7) or the intraparietal sulcus is classically reported in every PET or fMRI study that uses paired figurative stimuli and is believed by most authors to be responsible for the encoding of spatial relations, allocation of visual attention, and the integration of sensory and movement-related responses that contribute to the very act of mental rotation. The paired figurative stimulus paradigm also elicited activation of the frontal oculomotor field (BA 8). Because visual comparison of the stimuli is also required in the control condition, it appears that mental rotation of paired figures necessitates additional saccadic scanning of the visual images. This finding is in agreement with research on eye movements during mental rotation with paired stimuli (Just and Carpenter, 1985) and the neural model of mental rotation proposed by Cohen et al. (1996). It can, however, be argued that left parietal lobule activation in the present study is caused by the rotation of particularly the right picture, thus creating an attentional bias, making specificity for mental rotation itself hard to substantiate (Corbetta et al., 1993). Although a similar bias was available in the control task, this might not compensate for the increased right-ward shift of attention toward a rotated stimulus with occasional diversions to the left template image, as illustrated by the right prefrontal eye-field activation. A similar rightsided attention bias was present in several studies using a paired stimulus paradigm (and remained unclear in others), only one of which (the paired hands condition of the Kosslyn et al. (1998) study) also found parietal activation confined to the left hemisphere. Despite a right-sided attention bias, other studies found bilateral parietal activation (Cohen et al., 1996; Tagaris et al., 1996). Note also that single stimulus paradigms, where the lateralization of attention is eliminated, do not agree on hemispheric lateralization of mental rotation and have found parietal activation in the left hemisphere (Alivesatos and Petrides, 1997), the right hemisphere (Harris et al., 2000), or in both hemispheres (Tagaris et al., 1998). Nevertheless, future research should control for this potential confounding attention bias in paired stimuli designs by at least randomizing the position of the rotated stimulus.

Interestingly, the paired figurative stimulus paradigm also revealed a deactivation of the supplementary motor area (BA 6) in the medial part of the superior frontal gyrus. This area is intimately connected with 
the posterior parietal lobe and the motor cortex and is associated with grasping behavior (Kosslyn et al., 1998). Deactivation of this secondary motor cortex could suggest that a physical manipulation of the visual object is anticipated and that motor processes are triggered, but must be suppressed to comply with the requirements of the task, namely to mentally rotate the stimulus instead of actually turning it. The involvement of premotor and oculomotor regions in this task further suggests that motor processes may, at least to some degree, be an inherent part of every mental rotation and may explain the similar "physical" performance behavior found in different mental rotation paradigms. Premotor regions have been associated with the guidance of grasping behavior (Kosslyn et al., 1998) and the activation of oculomotor regions also (and independently) appeared in the single stimulus paradigm and may be involved in the visualization of movement (see further). The recruitment of predominantly higher-level or low-level motor processes appears to be strongly dependent on the specific task or the specific stimuli, and perhaps even on the specific cognitive approach of each individual subject (remember that in the Iwaki et al. (1999) study only three of six volunteers showed activation in their frontal motor cortices).

Comparison of all rotation tasks with all controls to reveal a common involvement of mental rotation in both tasks showed an activation of the right frontal oculomotor area (BA 8/32), again suggesting increased scanning of the images when mental rotation is required. A concurrent deactivation of the posterior part of the right superior temporal gyrus remains unexplained.

The results of the present study only partially confirm the first hypothesis. Different paradigms of mental rotation give rise to activation in quite different brain regions, but the activated areas do not constitute a "basic" mental rotation pattern sprinkled with additional activation depending on and reflecting task specificity. Although the use of a conservative technique in determining the regions of activation may have masked the underlying mental rotation pattern, our results indicate that task specificity and experimental design determine the most significant changes in cerebral blood flow between the mental rotation and the control task. This finding stresses the need for a meticulous description of the exact procedure, including stimulus presentation and response strategy, to reveal potentially hidden variables that might contribute to differences between studies. In addition, detailed introspection of the problem solving strategy used by each subject might reveal individual differences in task behavior that could explain some of the divergent findings.

With regard to cerebral lateralization, our results seem to suggest a primarily left hemispheric activation in rotation tasks that rely more heavily on low-level motor processes (Kosslyn et al., 1998). If this motorbased processing implies visualized manipulation of the stimuli, the left hemispheric lateralization in our right-handed subjects should not be unexpected (Iacoboni et al., 1998). In the paired figurative stimulus paradigm both hemispheres seem to be involved although the superior parietal area that is believed to be responsible for the mental rotation per se was significantly active in the left hemisphere only. This finding confirms the more intense left parietal activity during mental rotation of the Alivisatos and Petrides study (1997).

A direct comparison of both paradigms (global comparison) and of both mental rotation tasks separately revealed specific and focal activation of one task over the other, thereby confirming our second hypothesis. Compared to a paired stimuli paradigm, working with single alphanumeric stimuli revealed an unexpected activation of the oculomotor frontal region. We would expect activity in this region to be greater in the paired stimuli paradigm where two visual stimuli have to be perceptually compared leading to more eye-movements. Perhaps the BA 8/32 region is not only responsible for voluntary conjugate movements of the eyes, but is also involved in higher-level motor processing for the visualization of movement for example by imaging the eye-movements necessary to rotate the stimulus in an upright position. This might also contribute to the explanation of the significant activation of this BA 8/32 region when the control task(s) is/are subtracted from the rotation task(s) in the paired stimuli and combined paradigm respectively. In addition, working with alphanumeric stimuli activates the left middle to superior temporal gyrus. We suggest that this finding represents the activation of a memorized alphanumeric template against which the single mirror-imaged and/or rotated stimulus is compared. Several studies have located semantic memory in the temporal lobe (Dalla-Barba et al., 1998; Hodges et al., 1992; Maguire and Mummery, 1999; Ricci et al., 1999). The left hemispheric temporal lateralization in our right-handed subjects may reflect the symbolic character of the retrieved material by the language dominant left hemisphere. Comparison of mental rotation tasks only, showed additional activation for the single alphanumeric task in the left primary motor cortex that can be associated with a possible involvement of low-level motor processes in the mental manipulation of familiar stimuli. The paired figurative stimuli paradigm (global comparison) and rotation task additionally activated the primary visual cortex. This finding can be interpreted in terms of an additional demand on low-level visual processes for the accurate perception of and attention to more complex visual stimuli (Fink et al., 1996). It remains unclear why the precentral gyrus activation in the alphanumeric rotation is more superior after subtracting figures rotation than after sub- 
tracting the alphanumeric control condition. Simultaneous monitoring of subtle movements during different rotation paradigms might provide some clues to explain this observation.

It appears from these comparative data that similar paradigms differing only in stimulus material but designed to test for the same cognitive function in the same subjects, using the same imaging technology and methodology, may lead to significantly different areas of activation. One might conclude that the behavioral concept of mental rotation is not associated with a singular brain function, but includes a compilation of cognitive brain processes. Careful and methodic variations in the experimental design of cognitive paradigms may further reveal the contribution of different cortical areas to the complex function of mental rotation.

\section{REFERENCES}

Alivisatos, B., and Petrides, M. 1997. Functional activation of the human brain during mental rotation. Neuropsychologia 35: 111118 .

Bonda, E., Petrides, M., Frey, S., and Evans A. 1995. Neural correlates of mental transformations of the body-in-space. Proc. Natl. Acad. Sci. USA 92: 11180-11184.

Carpenter, P. A., Just, M. A., Keller, T. A., Eddy, W., and Thulborn, K. 1999. Graded functional activation in the visuospatial system with the amount of task demand. J. Cogn. Neurosci. 11: 9-24.

Cohen, M. S., Kosslyn, S. M., Breiter, H. C., DiGirolamo, G. J., Thompson, W. L., Anderson, A. K., Bookheimer, S. Y., Rosen, B. R., and Belliveau, J. W. 1996. Changes in cortical activity during mental rotation. A mapping study using functional MRI. Brain 119: $89-100$.

Cooper, L. A, and Shepard, R. N. 1973. The time required to prepare for a rotated stimulus. Mem. Cogn. 1: 246-250.

Corbetta, M., Miezin, F. M., Shulman, G. L., and Petersen, S. E. 1993. A PET study of visuospatial attention. J. Neurosci. 13: 1202-1226.

Dalla-Barba, G., Parlato, V., Jobert, A., Samson, Y., and Pappata, S. 1998. Cortical networks implicated in semantic and episodic memory: Common or unique? Cortex 34: 547-561.

d'Asseler, Y., Koole, M., Van Laere, K., Van De Walle, R., Van de Wiele, C., Lemahieu, I., and Dierckx, R. 1999. Evaluation of the accuracy of MR-SPECT and SPECT-SPECT coregistration using 8 different algorithms. Nucl. Med. Commun. 20: 659-669.

Deutsch, G., Bourbon, T., Papanicolaou, A. C., and Eisenberg, H. M. 1988. Visuospatial tasks compared via activation of regional cerebral blood flow. Neuropsychologia 26: 445-452.

Ditunno, P. L., and Mann, V. A. 1990. Right hemisphere specialization for mental rotation in normals and brain damaged subjects. Cortex 26: 177-188.

Fink, G. R., Halligan, P. W., Marshall, J. C., Frith, C. D., Frackowiak, R. S. J., and Dolan, R. J. 1996. Where in the brain does visual attention select the forest and the trees? Nature 382: 626-628.

Friston, K. J., Ashburner, J., Frith, C. D., Poline, J. B., Heather, J. D., and Frackowiak, R. S. J. 1995. Spatial registration and normalization of images. Hum. Brain Mapp. 2: 165-189.

Georgopoulos, A. P., and Pellizzer, G. 1995. The mental and the neural: Psychological and neural studies of mental rotation and memory scanning. Neuropsychologia 33: 1531-1547.
Harris, I. M., Egan, G. F., Sonkkila, C., Tochon-Danguy, H. J., Paxinos, G., and Watson, J. D. G. 2000. Selective right parietal lobe activation during mental rotation. A parametric PET study. Brain 123: $65-73$.

Hodges, J. R., Patterson, K., Oxbury, S., and Funnell, E. 1992. Semantic dementia. Progressive fluent aphasia with temporal lobe atrophy. Brain 115: 1783-806.

Iacoboni, M., Woods, R. P., and Mazziotta, J. C. 1998. Bimodal (auditory and visual) left frontoparietal circuitry for sensorimotor integration and sensorimotor learning. Brain 121: 2135-2143.

Inoue, M., Yoshino, A., Suzuki, A., Ogasawara, T., and Nomura, S. 1998. Topographic study of human event-related potentials using a task requiring mental rotation. Neurosci. Lett. 253: 107-110.

Iwaki, S., Ueno, S., Imada, T., and Tonoike, M. 1999. Dynamic cortical activation in mental image porcessing revealed by biomagnetic measurement. NeuroReport 10: 1793-1797.

Just, M. A., and Carpenter, P. A. 1985. Cognitive coordinate systems: Accounts of mental rotation and individual differences in spatial ability. Psychol. Rev. 92: 137-172.

Kosslyn, S. M. 1994. Image and Brain. MIT Press, Cambridge, MA.

Kosslyn, S. M., Digirolamo, G. J., Thompson, W. L., and Alpert, N. M. 1998. Mental rotation of objects versus hands: Neural mechanisms revealed by positron emission tomography. Psychophysiology 35: 151-161.

Maguire, E. A., and Mummery, C. J. 1999. Differential modulation of a common memory retrieval network revealed by positron emission tomography. Hippocampus 9: 54-61.

Metha, Z., and Newcombe, F. 1991. A role for the left hemisphere in spatial processing. Cortex 27: 153-167.

Parsons, L. M. 1987. Imagined spatial transformations of one's hand and feet. Cogn. Psychol. 19: 178-241.

Parsons, L. M. 1994. Temporal and kinematic properties of motor behavior reflected in mentally simulated action. J. Exp. Psychol. Hum. Perc. Perf. 20: 709-730.

Parsons, L. M., Fox, P. T., Downs, J. H., Glass, T., Hirsch, T. B., Martin, C. C., Jerabek, P. A., and Lancaster, J. L., 1995. Use of implicit motor imagery for visual shape discrimination as revealed by PET. Nature 375: 54-58.

Ricci, P. T., Zelkowicz, B. J., Nebes, R. D., Meltzer, C. C., Mintun, M. A., and Becker, J. T. 1999. Functional neuroanatomy of semantic memory: Recognition opf semantic associations. NeuroImage 9: 88-96.

Richter, W., Ugurbil, K., Georgopoulos, A., and Kim, S.-G. 1997. Time-resolved fMRI of mental rotation. NeuroReport 8: 3697-3702.

Rizzolati, G., Camarda, R., Fogassi, L., Gentilucci, M., Luppino, G., and Matelli, M. 1988. Functional organization of inferior area 6 in the macaque monkey. II. Area F5 and the control of distal movements. Exp. Brain Res. 71: 491-507.

Sakata, H., and Tiara, M. 1994. Parietal control of hand action. Curr. Opin. Neurobiol. 4: 847-856.

Sekiyama, K. 1982. The kinesthetic aspects of mental representation in the identification of left and right hands. Perc. Psychophys. 32: 89-95.

Shepard, R. N., and Cooper, L. A. 1982. Mental Images and Their Transformations. MIT Press, Cambridge, MA.

Shepard, R. N., and Metzler, J. 1971. Mental rotation of threedimensional objects. Science 171: 701-703.

Tagaris, G. A., Kim, S.-G., Strupp, J. P., Andersen, P., Ugurbil, K., and Georgopoulos, A. P. 1996. Quantitative relations between parietal activation and performance in mental rotation. NeuroReport 7: 773-776.

Tagaris, G. A., Kim, S.-G., Strupp, J. P., Andersen, P., Ugurbil, K., 
and Georgopoulos, A. P. 1997. Mental rotation studied by functional magnetic resonance imaging at high field (4 tesla): Performance and cortical activation. J. Cogn. Neurosci. 9: 419-432.

Tagaris, G. A., Richter, W., Kim, S.-G., Pellizzer, G., Andersen, P., Ugurbil, K., and Georgopoulos, A. P. 1998. Functional magnetic resonance imaging of mental rotation and memory scanning: $\mathrm{A}$ multidimensional scaling analysis of brain activation patterns. Brain Res. Rev. 26: 106-112.

Taira, M., Mine, D., Georgopoulos, A. P., Murata, A., and Sakata, H.
1990. Parietal cortex neurons of the monkey related to visual guidance of hand movements. Exp. Brain Res. 83: 29-36.

Talaraich, J., and Tournoux, P. 1988. Co-planar Stereotactic Atlas of the Human Brain. Thieme Medical Publishers, Stuttgart.

Wexler, M., Kosslyn, S. M., and Berthoz, A. 1998. Motor processes in mental rotation. Cognition 68: 77-94.

Zacks, J. Rypma, B., Gabrieli, J. D. E., Tversky, B., and Glover, G. H. 1999. Imagined transformations of bodies: An fMRI investigation. Neuropsychologia 37: 1029-1040. 